University of New Orleans

ScholarWorks@UNO

2-14-2007

\title{
Quarter-wave layers with $50 \%$ reflectance for obliquely incident unpolarized light
}

\author{
R. M.A. Azzam \\ University of New Orleans, razzam@uno.edu \\ F. F. Sudradjat
}

Follow this and additional works at: https://scholarworks.uno.edu/ee_facpubs

Part of the Electrical and Electronics Commons, and the Optics Commons

\section{Recommended Citation}

R. M. A. Azzam and F. F. Sudradjat, "Quarter-wave layers with $50 \%$ reflectance for obliquely incident unpolarized light," J. Opt. Soc. Am. A 24, 850-855 (2007)

This Article is brought to you for free and open access by the Department of Electrical Engineering at ScholarWorks@UNO. It has been accepted for inclusion in Electrical Engineering Faculty Publications by an authorized administrator of ScholarWorks@UNO. For more information, please contact scholarworks@uno.edu. 


\title{
Quarter-wave layers with $\mathbf{5 0} \%$ reflectance for obliquely incident unpolarized light
}

\author{
R. M. A. Azzam and F. F. Sudradjat \\ Department of Electrical Engineering, University of New Orleans, New Orleans, Louisiana 70148
}

Received June 15, 2006; accepted September 28, 2006;

posted October 20, 2006 (Doc. ID 71932); published February 14, 2007

\begin{abstract}
The conditions under which light interference in a transparent quarter-wave layer of refractive index $n_{1}$ on a transparent substrate of refractive index $n_{2}$ leads to $50 \%$ reflectance for incident unpolarized light at an angle $\varphi$ are determined. Two distinct solution branches are obtained that correspond to light reflection above and below the polarizing angle, $\varphi_{p}$, of zero reflection for $p$ polarization. The real $p$ and $s$ amplitude reflection coefficients have the same (negative) sign for the solution branch $\varphi>\varphi_{p}$ and have opposite signs for the solution branch $\varphi<\varphi_{p}$. Operation at $\varphi<\varphi_{p}$ is the basis of a 50\%-50\% beam splitter that divides an incident totally polarized light beam (with $p$ and $s$ components of equal intensity) into reflected and refracted beams of orthogonal polarizations [Opt. Lett. 31, $1525(2006)]$ and requires a film refractive index $n_{1} \geqslant(\sqrt{2}+1) \sqrt{n_{2}}$. A monochromatic design that uses a high-index $\mathrm{TiO}_{2}$ thin film on a low-index $\mathrm{MgF}_{2}$ substrate at $488 \mathrm{~nm}$ wavelength is presented as an example. (C) 2007 Optical Society of America

OCIS codes: $230.1360,260.5430,260.3160,160.4330$.
\end{abstract}

\section{INTRODUCTION}

In a recent letter ${ }^{1}$ it was shown that it is possible to split a monochromatic light beam into two beams of equal power and orthogonal polarizations by reflection and refraction at the planar surface of a dielectric substrate at an angle of incidence below the Brewster angle. This requires a substrate of high refractive index, $n_{2} \geqslant 3+2 \sqrt{2}$ $=5.8284$, e.g., PbTe in the IR. It was noted that a quarterwave layer of high refractive index, which is deposited on a substrate of low refractive index, can also be used to accomplish the same novel beam splitting function. This thin-film coating design is now presented in this paper. For a succinct account of different types of beam splitters, see, e.g., the review by Dobrowolski. ${ }^{2}$

In Section 2 an analytical design procedure is presented for achieving 50\% reflectance of unpolarized (or randomly polarized) light that is obliquely incident on a transparent quarter-wave coating on a transparent substrate. The algorithm developed in Section 2 is applied in Subsection 3.A to the special case of a vanishing substrate $\left(n_{2}=1\right)$, i.e., an unsupported quarter-wave pellicle. It is also applied to quarter-wave coatings of varying refractive index $n_{1}$ on a (glass or plastic) substrate of refractive index $n_{2}=1.5$ in Subsection 3.B.

In Section 4 a beam splitter design that uses a $\mathrm{TiO}_{2}$ high-index quarter-wave coating on a low-index $\mathrm{MgF}_{2}$ substrate for $488 \mathrm{~nm}$ (Ar-ion-laser) light is considered as a specific example. An error analysis shows the effect of $\pm 5^{\circ}$ shifts in the angle of incidence and of $\pm 5 \%$ shifts in wavelength or film thickness on the performance of this beam splitter. Section 5 gives a brief summary of the paper.

\section{QUARTER-WAVE LAYERS THAT REFLECT $50 \%$ OF UNPOLARIZED LIGHT AT OBLIQUE INCIDENCE: ANALYTICAL TREATMENT}

For light reflection in air $\left(n_{0}=1\right)$ by a transparent layer of quarter-wave optical thickness (equal to half the filmthickness period) and refractive index $n_{1}$ on a transparent substrate of refractive index $n_{2}$, the amplitude reflection coefficients of the $p$ and $s$ polarizations at oblique incidence at an angle $\varphi$ are real and are given $b^{3,4}$

$$
\begin{aligned}
& R_{s}=\frac{S_{0} S_{2}-S_{1}^{2}}{S_{0} S_{2}+S_{1}^{2}}, \\
& R_{p}=\frac{n_{1}{ }^{4} S_{0} S_{2}-n_{2}{ }^{2} S_{1}^{2}}{n_{1}{ }^{4} S_{0} S_{2}+n_{2}{ }^{2}{S_{1}}^{2}},
\end{aligned}
$$

where

$$
\begin{aligned}
S_{i} & =\left(n_{i}^{2}-u\right)^{1 / 2}, \quad i=0,1,2, \\
u & =\sin ^{2} \varphi .
\end{aligned}
$$

All materials are assumed to be optically isotropic and are separated by parallel plane boundaries.

In terms of the quantity

$$
P=S_{0} S_{2} / S_{1}^{2}
$$

Eqs. (1) and (2) are rewritten as 


$$
R_{s}=\frac{P-1}{P+1}, \quad R_{p}=\frac{n_{1}{ }^{4} P-n_{2}{ }^{2}}{n_{1}{ }^{4} P+n_{2}{ }^{2}} .
$$

To achieve $50 \%$ intensity reflectance for incident unpolarized light, the amplitude reflection coefficients of the $p$ and $s$ polarizations must satisfy the following condition:

$$
R_{p}^{2}+R_{s}^{2}=1 \text {. }
$$

If Eqs. (6) are substituted into Eq. (7), a quartic equation in $P$ is obtained,

$$
a_{4} P^{4}+a_{3} P^{3}+a_{2} P^{2}+a_{1} P+a_{0}=0,
$$

with coefficients given by

$$
\begin{aligned}
& a_{4}=n_{1}{ }^{8}, \\
& a_{3}=-2 n_{1}{ }^{4}\left(n_{1}{ }^{4}+n_{2}{ }^{2}\right), \\
& a_{2}=n_{1}{ }^{4}\left(n_{1}{ }^{4}-12 n_{2}{ }^{2}\right)+n_{2}{ }^{4}, \\
& a_{1}=-2 n_{2}{ }^{2}\left(n_{1}{ }^{4}+n_{2}{ }^{2}\right), \\
& a_{0}=n_{2}{ }^{4} .
\end{aligned}
$$

For a high-index transparent film on a low-index transparent substrate it is apparent from Eqs. (3)-(5) that $P$ is real and positive and in the range $0<P<1$, hence, only roots of Eq. (8) that satisfy this condition are accepted.

From Eqs. (3)-(5) we also obtain

$$
P^{2}=(1-u)\left(n_{2}{ }^{2}-u\right) /\left(n_{1}{ }^{2}-u\right)^{2} .
$$

Equation (10) can be rewritten as a quadratic equation in $u$ :

$$
b_{2} u^{2}+b_{1} u+b_{0}=0,
$$

with coefficients given by

$$
\begin{aligned}
& b_{2}=P^{2}-1, \\
& b_{1}=\left(n_{2}{ }^{2}+1\right)-2 n_{1}{ }^{2} P^{2}, \\
& b_{0}=n_{1}{ }^{4} P^{2}-n_{2}{ }^{2} .
\end{aligned}
$$

The only acceptable roots of Eq. (11) are those for which $0 \leqslant u<1$. Finally, the angle of incidence $\varphi$ at which the $50 \%$ reflectance for incident unpolarized light is achieved, for given $n_{1}$ and $n_{2}$, is given by

$$
\varphi=\arcsin \left(u^{1 / 2}\right) .
$$

\section{QUARTER-WAVE LAYERS THAT REFLECT 50\% OF UNPOLARIZED LIGHT AT OBLIQUE INCIDENCE: NUMERICAL RESULTS}

\section{A. Case of a Vanishing Substrate, $n_{2}=1$}

The algorithm of Section 2 is applied to determine the constraint between $n_{1}$ and $\varphi$ such that Eq. (7) is satisfied when $n_{2}=1$ (i.e., for an unsupported quarter-wave layer or pellicle), by assigning values to $n_{1}$ between 1 and 6 in steps of 0.01 and solving for the corresponding values of $\varphi$.

Figure 1 shows the two solution branches that we obtain: a high-angle branch (HAB), or solution 1, which is represented by the continuous line, and a low-angle branch (LAB), or solution 2 , which is represented by the dashed line. The dash-dot curve in the middle represents the polarizing (Brewster) angle, $\varphi_{p}=\varphi_{B}=\tan ^{-1} n_{1}$.

Figures 2 and 3 show the real amplitude reflection coefficients $R_{p}$ and $R_{s}$ that are associated with the HAB and $\mathrm{LAB}$, respectively, as functions of the film refractive index $n_{1} . R_{p}$ and $R_{s}$ are both negative for the HAB, Fig. 2, and have opposite signs $\left(R_{p}>0, R_{s}<0\right)$ for the LAB, Fig. 3.

Figure 4 is a plot of $R_{p}$ versus $R_{s}$ and shows two separate arcs of the unit circle, Eq. (7), in the third and fourth quadrants, respectively. Points A and B correspond to the similarly marked points in Fig. 1.

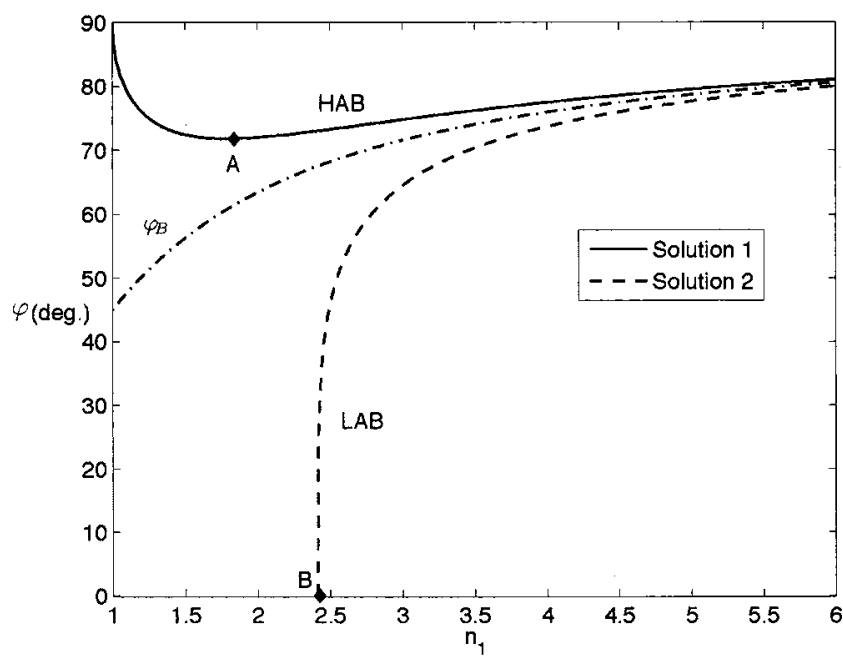

Fig. 1. Angle of incidence $\varphi$ versus film refractive index $n_{1}$ such that $50 \%$ of incident unpolarized light is reflected by a quarterwave pellicle. The continuous and dashed curves represent two independent solution branches. The middle curve gives the Brewster angle as a function of $n_{1}, \varphi_{B}=\tan ^{-1} n_{1}$.

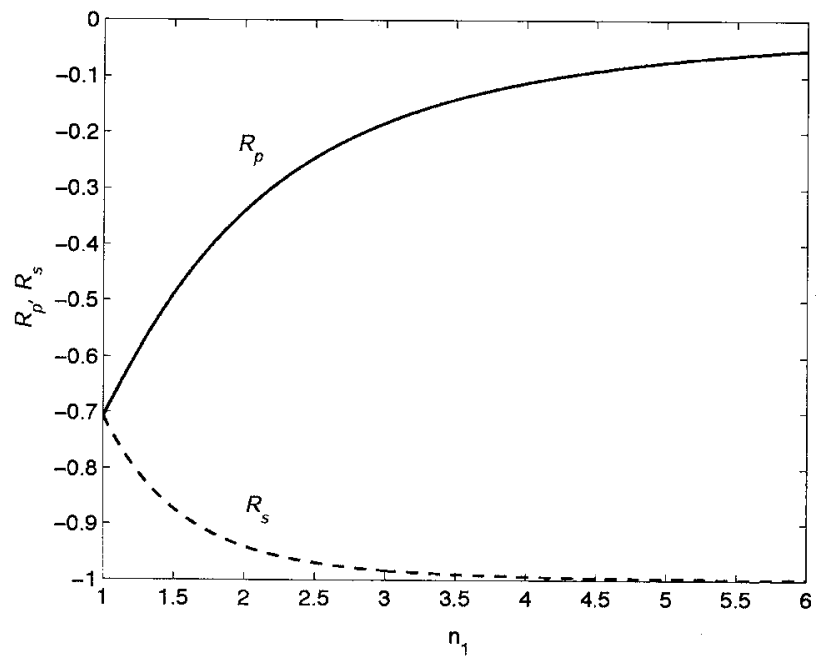

Fig. 2. Amplitude reflection coefficients $R_{p}$ and $R_{s}$ that are associated with the HAB of Fig. 1 plotted as functions of the film refractive index $n_{1}$. 


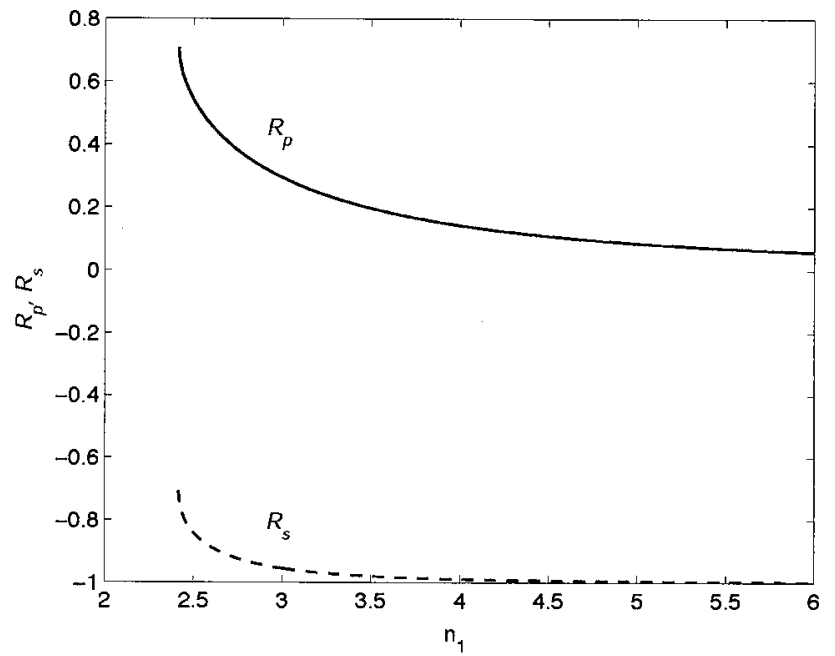

Fig. 3. Amplitude reflection coefficients $R_{p}$ and $R_{s}$ that are associated with the LAB of Fig. 1 plotted as functions of the film refractive index $n_{1}$.

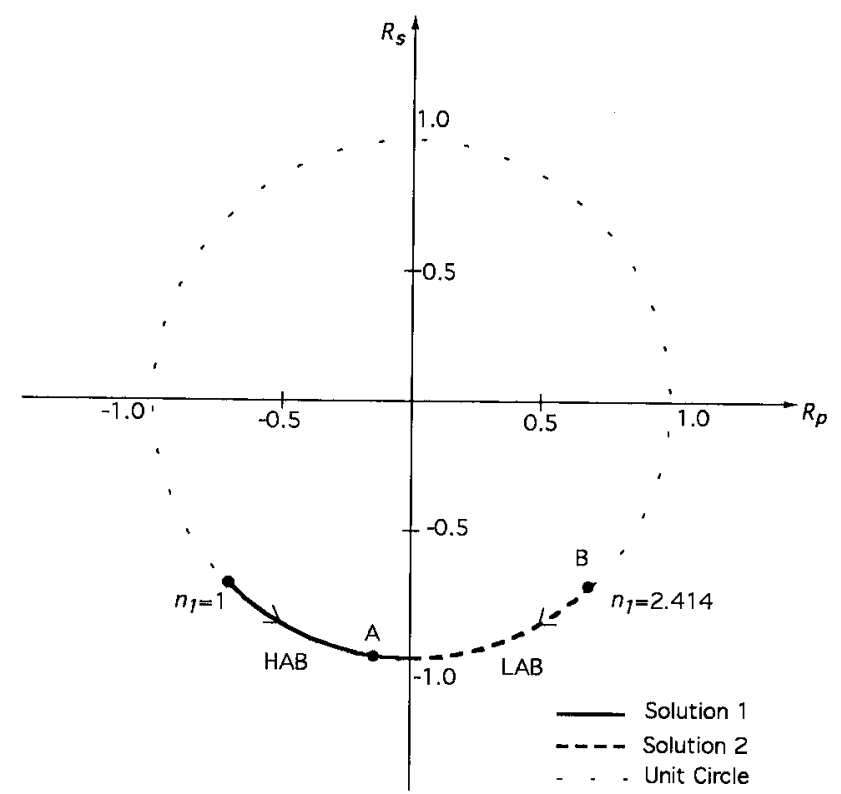

Fig. 4. Plot of $R_{p}$ versus $R_{s}$ associated with the high- and lowangle branches (Figs. 2 and 3 ) yields two arcs of the unit circle in the third and fourth quadrants, respectively. The arrows indicate the direction of increasing $n_{1}$, and points $\mathrm{A}$ and $\mathrm{B}$ correspond to the similarly marked points in Fig. 1.

Figures 5 and 6 show the intensity reflectances $R_{p}{ }^{2}$ and $R_{\mathrm{s}}{ }^{2}$ for the HAB and LAB, respectively, as functions of $n_{1}$. The average value of $R_{p}{ }^{2}$ and $R_{s}^{2}$ is constant (=0.5), independent of $n_{1}$ as is required by Eq. (7).

An interesting feature of Fig. 1 is that $\varphi$ of the HAB reaches a minimum, $\varphi_{\min }=71.8225^{\circ}$, at point A where $n_{1}$ $=1.78$. Therefore, a pellicle of 1.78 refractive index and quarter-wave optical thickness (or an odd integral multiple thereof) functions as a $50 \%-50 \%$ BS for incident unpolarized light at $\approx 72^{\circ}$ angle of incidence. The starting point $\mathrm{B}$ of the LAB in Fig. 1 is at $\varphi=0$ and corresponds to $n_{1}=\sqrt{2}+1=2.414$. Film materials that have this refractive index include diamond in the visible and $\mathrm{ZnSe}$ in the IR. $^{5,6}$
B. General Case of $n_{2}>1$

For an arbitrary substrate of refractive index $n_{2}$, the starting value of $n_{1}$ for the LAB is obtained by noting that at $\varphi=0, R_{p}{ }^{2}=R_{s}{ }^{2}=1 / 2$. It follows from Eqs. (6) that

$$
\begin{aligned}
R_{s} & =(P-1) /(P+1)=-1 / \sqrt{2}, \\
P & =(\sqrt{2}-1)^{2} .
\end{aligned}
$$

If $u=0$ is substituted in Eq. (10), we get

$$
n_{1}=\left(n_{2} / P\right)^{1 / 2} \text {. }
$$

When Eqs. (15) and (16) are combined, we obtain

$$
n_{1}=(\sqrt{2}+1) \sqrt{n_{2}} \text {. }
$$

As a specific example we take quarter-wave layers on a (glass or plastic) substrate with $n_{2}=1.5$. Figure 7 shows the two solution branches that we obtain using the algorithm of Sec. 2. When compared with Fig. 1 (for $n_{2}=1$ ), the HAB in Fig. 7 is displaced upward to higher angles,

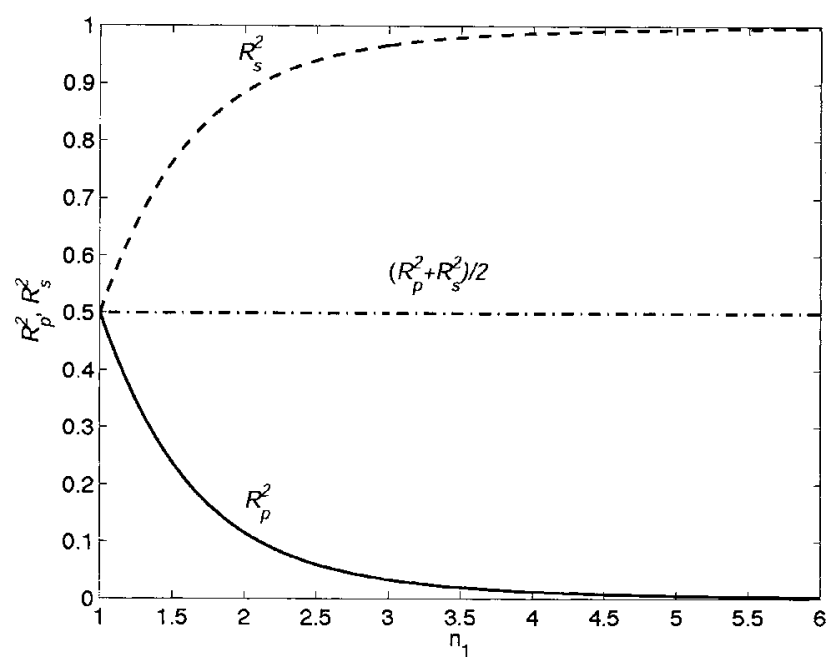

Fig. 5. Intensity reflectances $R_{p}{ }^{2}$ and $R_{s}{ }^{2}$ that are associated with the HAB of Fig. 1 plotted as functions of the film refractive index $n_{1}$. The average reflectance, $\left(R_{p}{ }^{2}+R_{s}{ }^{2}\right) / 2$, is constant at 0.5 .

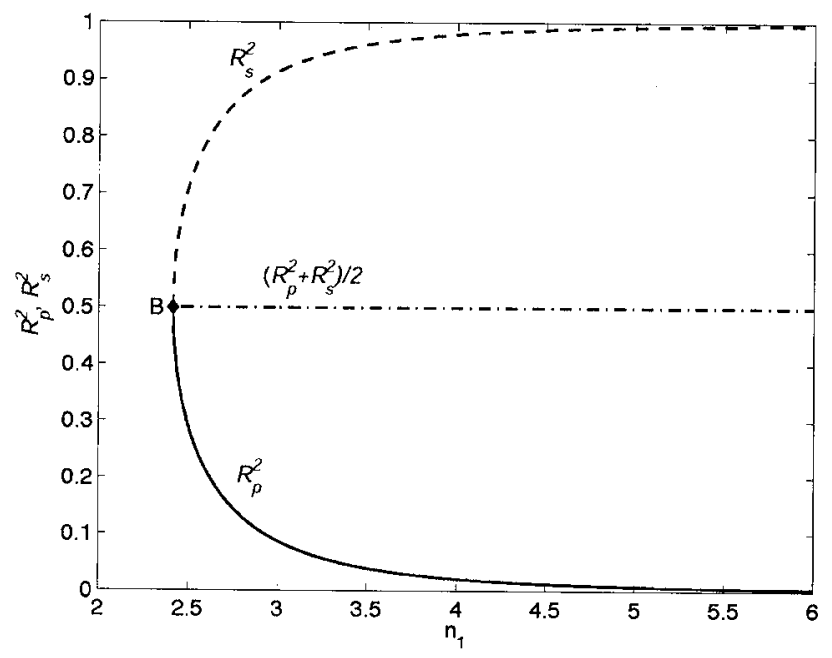

Fig. 6. Intensity reflectances $R_{p}{ }^{2}$ and $R_{s}{ }^{2}$ that are associated with the LAB of Fig. 1 plotted as functions of the film refractive index $n_{1}$. The average reflectance, $\left(R_{p}{ }^{2}+R_{s}{ }^{2}\right) / 2$, is constant at 0.5 . 


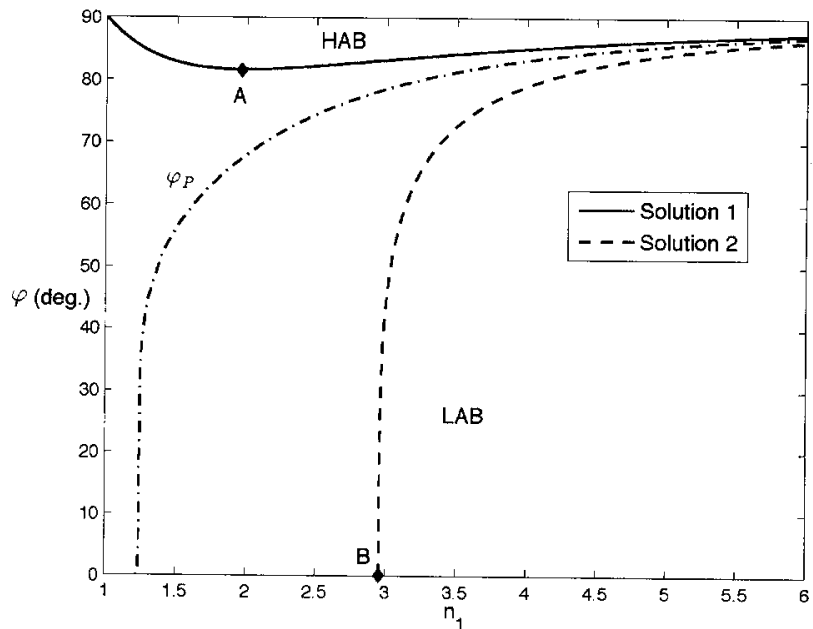

Fig. 7. Angle of incidence $\varphi$ versus the refractive index $n_{1}$ of a transparent quarter-wave coating on a transparent substrate $\left(n_{2}=1.5\right)$ that reflects $50 \%$ of incident unpolarized light. The continuous and dashed curves represent two distinct solution branches. The middle dash-dot curve gives the polarizing angle $\varphi_{p}$ as a function of $n_{1}$ as obtained from Eqs. (19) and (20).

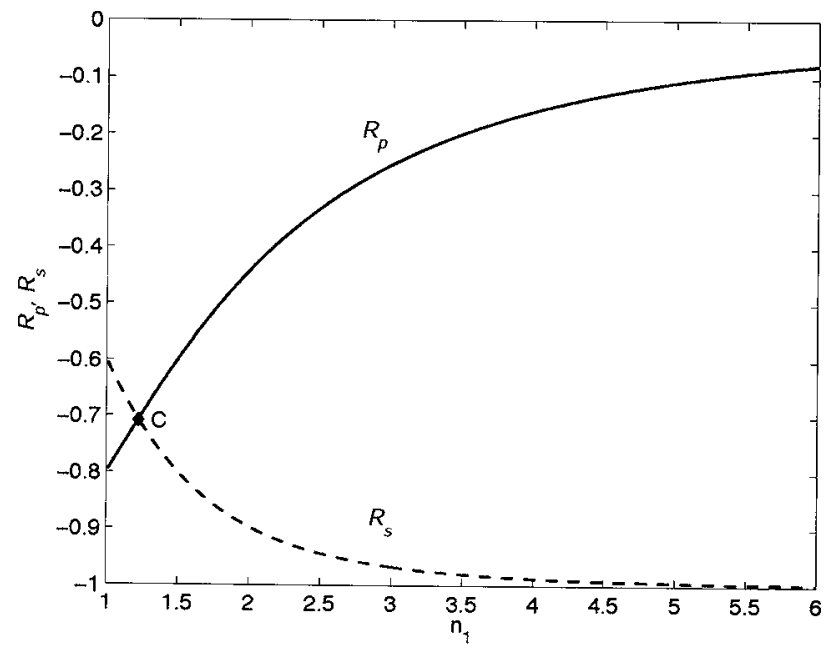

Fig. 8. Amplitude reflection coefficients $R_{p}$ and $R_{s}$ that are associated with the HAB of Fig. 7 plotted as functions of the film refractive index $n_{1}$. The significance of the point of intersection $\mathrm{C}$ is discussed in the text.

and its minimum at point $\mathrm{A}$ now occurs at $\varphi_{\min }$ $=81.6208^{\circ}, n_{1}=1.98$. The starting value $n_{1}=2.9568$ of the $\mathrm{LAB}$ at point $\mathrm{B}$ is predicted by Eq. (17).

In Fig. 7 the middle (dash-dot) curve gives the polarizing angle $\varphi_{p}$ of the film-substrate system at which $R_{p}=0$ as a function of the film refractive index $n_{1}$ with $n_{2}=1.5$. To calculate the polarizing angle $\varphi_{p}$ we note from Eqs. (6) that $R_{p}=0$ if

$$
P=n_{2}{ }^{2} / n_{1}{ }^{4} \text {. }
$$

Substitution of $P$ from Eq. (18) into Eq. (10) gives a quadratic equation,

$$
c_{2} u^{2}+c_{1} u+c_{0}=0,
$$

in $u=\sin ^{2} \varphi_{p}$ with coefficients given by

$$
c_{2}=n_{1}^{8}-n_{2}^{8},
$$

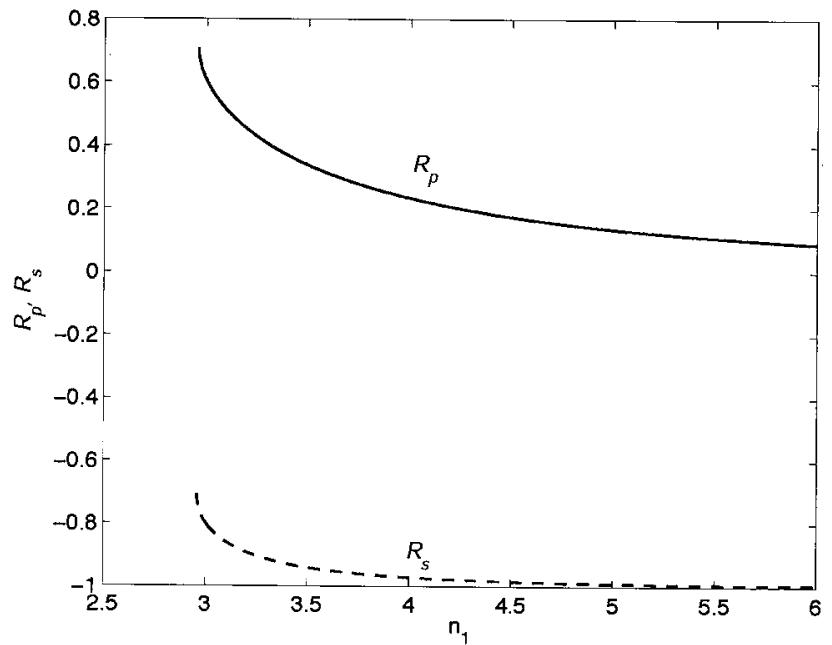

Fig. 9. Amplitude reflection coefficients $R_{p}$ and $R_{s}$ that are associated with the LAB of Fig. 7 plotted as functions of the film refractive index $n_{1}$.

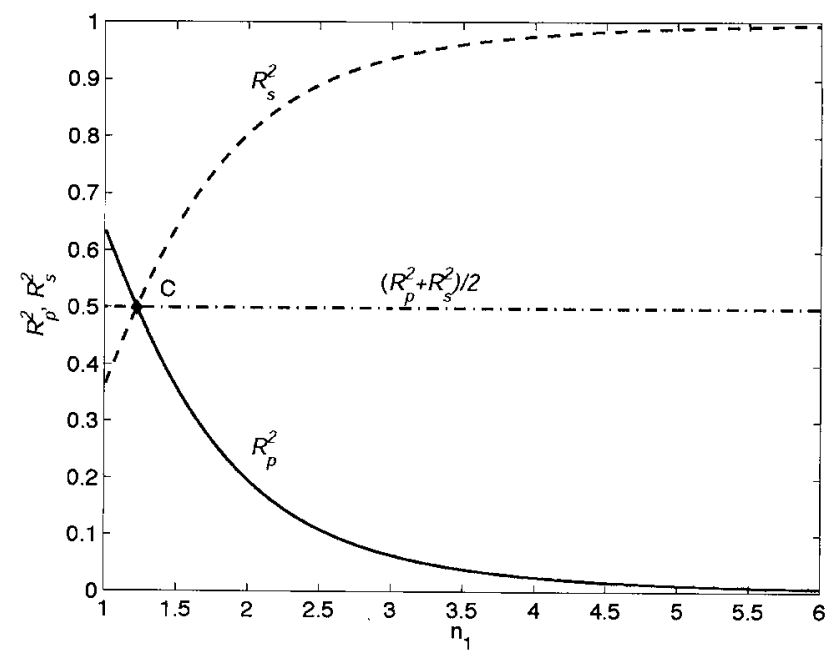

Fig. 10. Intensity reflectances $R_{p}{ }^{2}$ and $R_{s}{ }^{2}$ that are associated with the HAB of Fig. 7 plotted as functions of the film refractive index $n_{1}$. The average reflectance, $\left(R_{p}{ }^{2}+R_{s}{ }^{2}\right) / 2$, is constant at 0.5 .

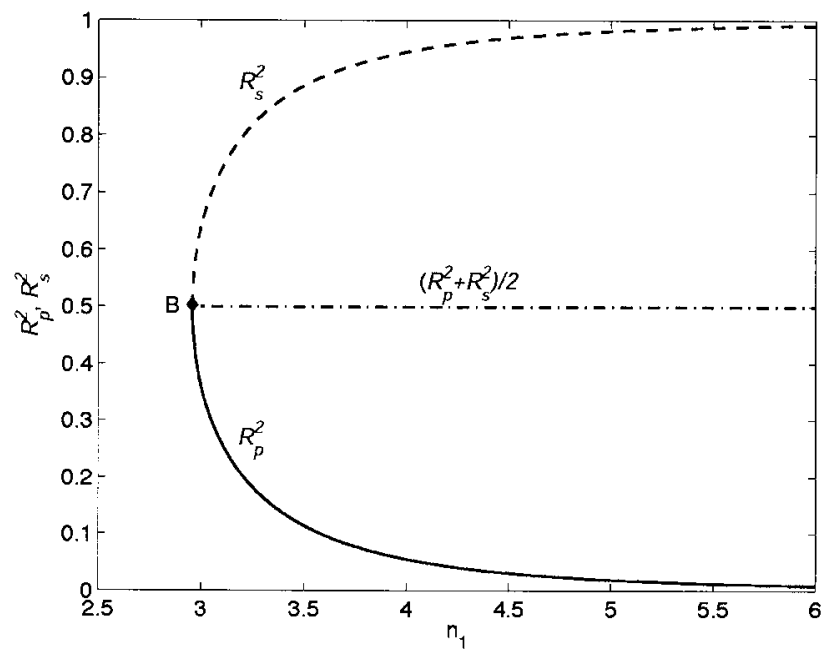

Fig. 11. Intensity reflectances $R_{p}{ }^{2}$ and $R_{s}{ }^{2}$ that are associated with the LAB of Fig. 7 plotted as functions of the film refractive index $n_{1}$. The average reflectance, $\left(R_{p}{ }^{2}+R_{s}{ }^{2}\right) / 2$, is constant at 0.5 . 

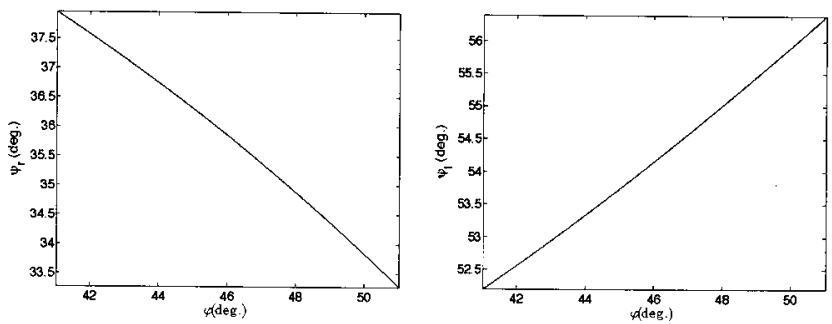

(a)

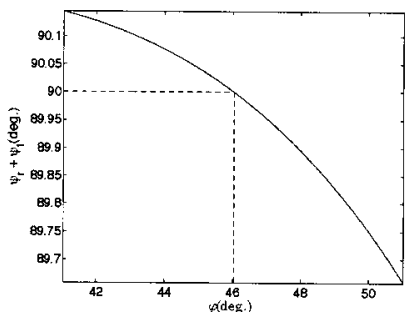

(c)

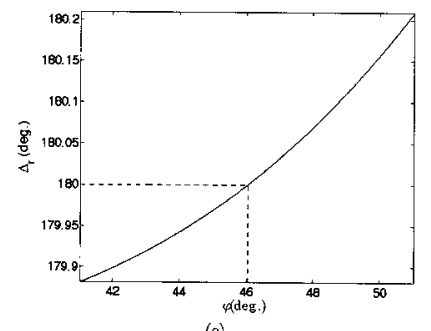

(e)

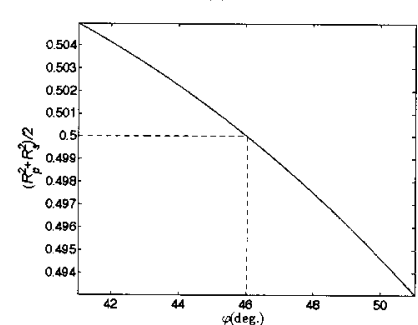

(d)

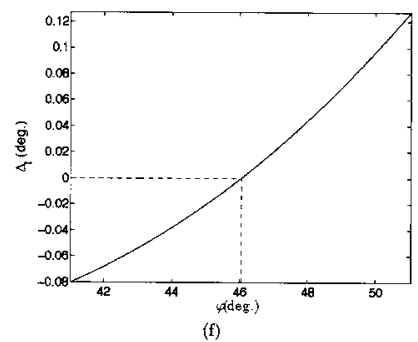

Fig. 12. Average reflectance $\left(R_{p}{ }^{2}+R_{s}{ }^{2}\right) / 2$ and ellipsometric parameters $\psi_{r}, \psi_{t}, \Delta_{r}, \Delta_{t}$ as functions of the angle of incidence $\varphi$ for a beam splitter that consists of a quarter-wave $(43.51 \mathrm{~nm})$ thin film of $\mathrm{TiO}_{2}$ on a $\mathrm{MgF}_{2}$ substrate at $488 \mathrm{~nm}$ wavelength. The angle of incidence is varied by $\pm 5^{\circ}$ around the design angle $\varphi$ $=46.04^{\circ}$.

$$
\begin{aligned}
& c_{1}=2 n_{1}{ }^{2} n_{2}{ }^{4}-n_{1}{ }^{8}\left(n_{2}{ }^{2}+1\right), \\
& c_{0}=n_{1}{ }^{4} n_{2}{ }^{2}\left(n_{1}{ }^{4}-n_{2}{ }^{2}\right) .
\end{aligned}
$$

Figures 8 and 9 show the amplitude refection coefficients $R_{p}$ and $R_{s}$ as functions of $n_{1}$ for the HAB and LAB of Fig. 7, respectively. Again, $R_{p}$ and $R_{s}$ are both negative for the HAB, Fig. 8, and have opposite signs $\left(R_{p}>0, R_{s}\right.$ $<0)$ for the LAB, Fig. 9.

The point of intersection $\mathrm{C}$ of the curves of $R_{p}$ and $R_{s}$ in Fig. 8 is located at

$$
R_{p}=R_{s}=-1 / \sqrt{2}=-0.7071, \quad n_{1}=\sqrt{n_{2}}=1.2247 .
$$

This corresponds to a polarization-preserving (or polarization-independent) $50 \%-50 \%$ beam splitter in both reflection and transmission. ${ }^{3}$

The associated intensity reflections $R_{p}{ }^{2}$ and $R_{s}{ }^{2}$ as functions of $n_{1}$ are shown in Figs. 10 and 11 for the HAB and LAB, respectively. Again, the average of $R_{p}{ }^{2}$ and $R_{s}{ }^{2}$ is constant $(=0.5)$ independent of $n_{1}$ as required by Eq. (7).

\section{BEAM SPLITTER FOR PRODUCING REFLECTED AND TRANSMITTED BEAMS OF EQUAL POWER AND ORTHOGONAL POLARIZATIONS IN THE VISIBLE}

Consider a high-index $\left(n_{1}=2.895\right)$ thin film of $\mathrm{TiO}_{2}$ on a low-index $\left(n_{2}=1.386\right) \mathrm{MgF}_{2}$ substrate at wavelength $\lambda$

$=488 \mathrm{~nm}$. (Because we assume isotropic phases, the refractive indices are taken as averages of the ordinary and extraordinary indices $n_{0}$ and $n_{\mathrm{e}}$ published in Ref. 6.) By use of the algorithm of Section 2, the operating angle of incidence $\varphi=46.037^{\circ}$ is obtained. The film-thickness period $D_{1}$ and quarter-wave metric film thickness $d_{1}$ are determined by

$$
\begin{aligned}
& D_{1}=(\lambda / 2)\left(n_{1}^{2}-\sin ^{2} \varphi\right)^{-1 / 2}, \\
& d_{1}=D_{1} / 2,
\end{aligned}
$$

which give $D_{1}=87.02 \mathrm{~nm}$ and $d_{1}=43.51 \mathrm{~nm}$. Under the ideal operating conditions, the average reflectance for incident unpolarized light is $1 / 2$ and the reflection and transmission ellipsometric parameters ${ }^{4}$ satisfy the following relations: ${ }^{1,7}$

$$
\begin{aligned}
& \Delta_{r}=\pi, \Delta_{t}=0, \\
& \psi_{r}+\psi_{t}=\pi / 2 .
\end{aligned}
$$

To couple the refracted light out of the substrate into air, a prismatic substrate with prism angle equal to the angle of refraction $\left(31.287^{\circ}\right)$ can be used, so that light leaves the prism normal to its exit face which is antireflection coated. $^{8}$

When the wavelength of light and metric film thickness are kept constant, and the angle of incidence is varied by up to $\pm 5^{\circ}$, the average reflectance and ellipsometric pa-
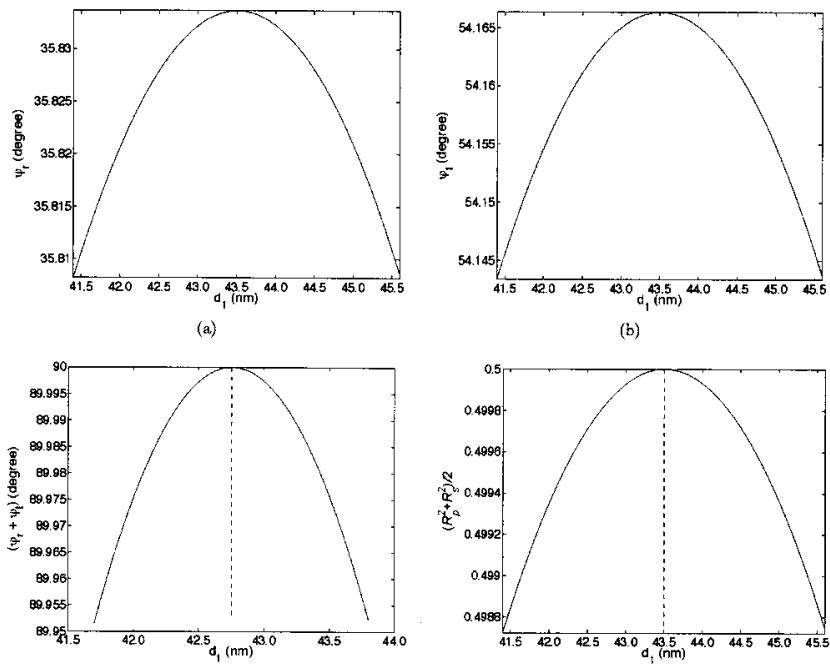

(b)

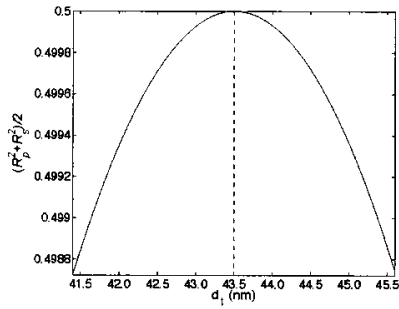

(d)

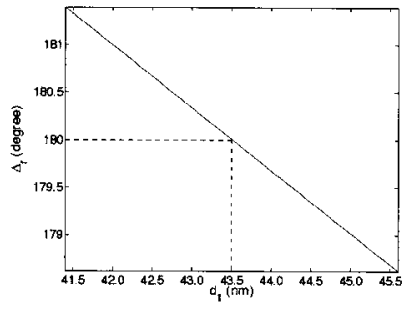

(e)

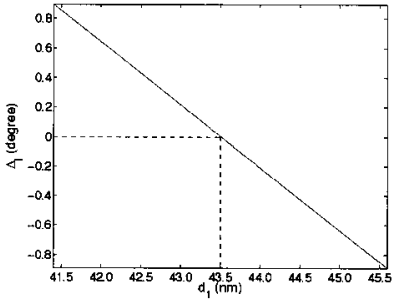

(f)

Fig. 13. Average reflectance $\left(R_{p}{ }^{2}+R_{s}{ }^{2}\right) / 2$ and ellipsometric parameters $\psi_{r}, \psi_{t}, \Delta_{r}, \Delta_{t}$ of a beam splitter as functions of the thickness $d_{1}$ of a $\mathrm{TiO}_{2}$ film on a $\mathrm{MgF}_{2}$ substrate at $\lambda=488 \mathrm{~nm}$ and $\varphi$ $=46.04^{\circ}$. The film thickness is varied by $\pm 5 \%$ around the design value of $43.5 \mathrm{~nm}$. 


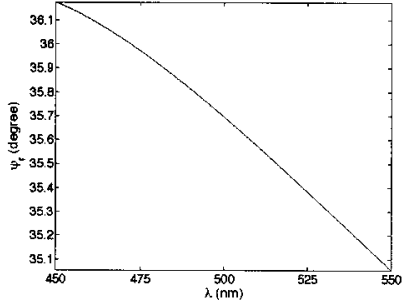

(a)

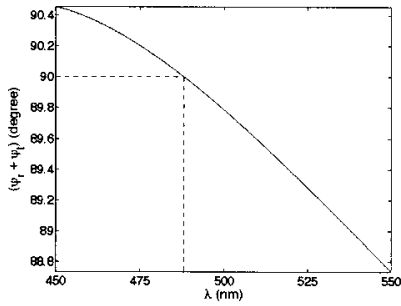

(c)

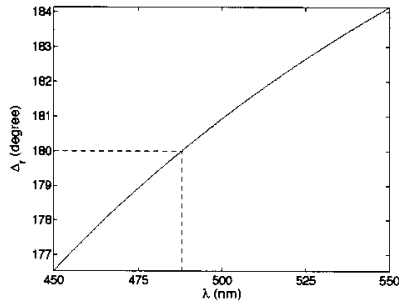

(e)

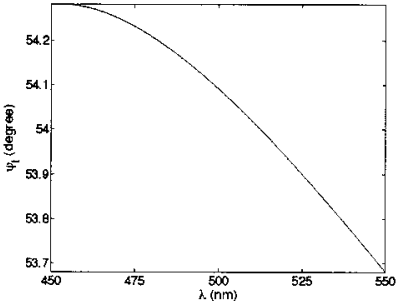

(b)

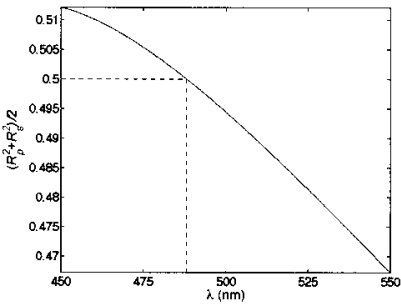

(d)

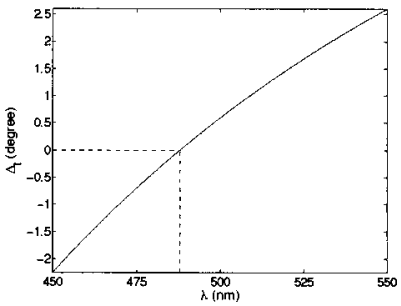

(f)
Fig. 14. Average reflectance $\left(R_{p}{ }^{2}+R_{s}{ }^{2}\right) / 2$ and ellipsometric parameters $\psi_{r}, \psi_{t}, \Delta_{r}, \Delta_{t}$ as functions of the wavelength $\lambda$ (in nanometers) for a beam splitter that consists of a $43.51 \mathrm{~nm} \mathrm{TiO}_{2}$ thin film on a $\mathrm{MgF}_{2}$ substrate at an angle of incidence of $\varphi=46.04^{\circ}$. The wavelength $\lambda$ is changed by $\pm 5 \%$ around $488 \mathrm{~nm}$.

rameters change in the manner shown in Fig. 12. From Fig. 12, it is apparent that the ideal conditions of Eqs. (23) are nearly maintained; hence, this novel beam splitter is tolerant to small angle-of-incidence errors.

If the wavelength of light and angle of incidence are fixed, but the film thickness is varied by $\pm 5 \%$ around its design value $\left(d_{1}=43.51 \mathrm{~nm}\right)$, the average reflectance and ellipsometric parameters change in the manner shown in Fig. 13. Again, the ideal conditions of Eqs. (23) are approximately satisfied, to within a small error, and the coating design is not overly sensitive to small filmthickness errors around the quarter-wave thickness.

Finally, when the angle of incidence and metric thickness of the coating are kept constant, and the wavelength is shifted by $\pm 5 \%$ around $\lambda=488 \mathrm{~nm}$, the average reflectance and ellipsometric parameters change in the manner shown in Fig. 14. In Fig. 14, the deviations from the ideal conditions of Eqs. (23) are more pronounced; hence, the beam splitter is not considered achromatic.

\section{SUMMARY}

A transparent layer of quarter-wave optical thickness, a pellicle or a coating deposited on a transparent substrate, reflects $50 \%$ of incident unpolarized light under certain conditions that are completely determined. Two distinct solution branches are obtained that correspond to incidence below and above the $p$-suppressing polarizing angle of the film-substrate system. Operation below the polarizing angle makes possible a novel thin-film beam splitter $^{1}$ that divides incident totally polarized light into reflected and refracted beams of equal power ( $50 \%$ each) and orthogonal polarizations. The performance of one such device that uses a high-index $\mathrm{TiO}_{2}$ quarter-wave coating on a low-index $\mathrm{MgF}_{2}$ substrate at $488 \mathrm{~nm}$ wavelength is presented.

R.M.A. Azzam’s e-mail address is razzam@uno.edu.

\section{REFERENCES}

1. R. M. A. Azzam, "Dividing a light beam into two beams of orthogonal polarizations by reflection and refraction at a dielectric surface," Opt. Lett. 31, 1525-1527 (2006).

2. J. A. Dobrowolski, "Optical properties of films and coatings," in Handbook of Optics, M. Bass, E. W. Van Stryland, D. R. Williams, and W. L. Wolfe, eds. (McGrawHill, 1995), Vol. II, Chap. 42.

3. R. M. A. Azzam, "Simultaneous reflection and refraction of light without change of polarization by a single-layercoated dielectric surface," Opt. Lett. 10, 107-109 (1985).

4. R. M. A. Azzam and N. M. Bashara, Ellipsometry and Polarized Light (North-Holland, 1987).

5. Handbook of Optical Constants of Solids, E. D. Palik, ed. (Academic, 1985).

6. W. J. Tropf, M. E. Thomas, and T. J. Harris, "Optical properties of crystals and glasses," in Handbook of Optics, M. Bass, E. W. Van Stryland, D. R. Williams, and W. L. Wolfe, eds. (McGraw-Hill, 1995), Vol. II, Chap. 33.

7. R. M. A. Azzam and A. De, "Optimal beam splitters for the division-of-amplitude photopolarimeter," J. Opt. Soc. Am. A 20, 955-958 (2003).

8. R. M. A. Azzam, "Variable-reflectance thin-film polarization-independent beam splitters for 0.6328 and $10.6 \mu \mathrm{m}$ laser light," Opt. Lett. 10, 110-112 (1985). 